University of Nebraska - Lincoln

DigitalCommons@University of Nebraska - Lincoln

1985

\title{
Contrasts Among Bidirectional Reflectance of Leaves, Canopies, and Soils
}

Elizabeth A. Walter-Shea

University of Nebraska - Lincoln, ewalter-shea1@unl.edu

Follow this and additional works at: https://digitalcommons.unl.edu/natrespapers

Part of the Natural Resources and Conservation Commons, Natural Resources Management and Policy Commons, and the Other Environmental Sciences Commons

Walter-Shea, Elizabeth A., "Contrasts Among Bidirectional Reflectance of Leaves, Canopies, and Soils" (1985). Papers in Natural Resources. 1174.

https://digitalcommons.unl.edu/natrespapers/1174

This Article is brought to you for free and open access by the Natural Resources, School of at DigitalCommons@University of Nebraska - Lincoln. It has been accepted for inclusion in Papers in Natural Resources by an authorized administrator of DigitalCommons@University of Nebraska - Lincoln. 


\title{
Contrasts Among Bidirectional Reflectance of Leaves, Canopies, and Soils
}

\author{
JOHN M. NORMAN, JON M. WELLES, AND ELIZABETH A. WALTER
}

\begin{abstract}
The apparent brightness of a natural surface depends on the characteristics and direction of incident radiation, surface radiative properties, and the direction from which the surface is viewed. The bidirectional reflectance distribution functions (BRDF) for soils, vegetation canopies, and individual leaves have common features that arise from an anisotropic diffuse scattered component as well as specular reflections from irregular surfaces. The diff use scattered component, however, tends to dominate canopy and soil BRDF's where as specular reflection tends to dominate leaf BRDF's. In this paper, simple models are presented for predicting soil and canopy BRDF's, and predicted results are compared with some measurements. This effort represents an attempt to illustrate the important features that cause observed BRDF's. The results of the models are compared with a simple threecoefficient empirical equation that may be easier to invert than the causitive models so that radiation measurements can be used to obtain surface features. Some BRDF measurements from corn and soybean leaves provide a contrast for canopy and soil distributions, and also provide needed leaf properties that are important to vegetation canopy models. The knowledge of soil, canopy, and leaf BRDF's is combined into a single model, called Cupid, to predict the resultant BRDF of complex natural surfaces.
\end{abstract}

\section{INTRODUCTION}

$\mathrm{T}$ HE APPARENT brightness of a natural surface depends on the characteristics and direction of the incident radiation, surface radiative properties, and the direction from which the surface is viewed. Although the characteristics and direction of solar radiation incident on natural surfaces is quite well understood, the dependence of surface radiative properties on well-known physical characteristics remains to be established for most typical natural surfaces. Understanding the relation between remote radiation measurements and vegetation characteristics is essential if we are to use remote sensing as a vegetation monitoring tool. Most of the radiation measurements, which have been made above natural surfaces such as soils or canopies, have consisted of only nadir reflectance factors. Although such nadir viewing measurements have the advantage of simplicity, the amount of information that can be inferred about the surface is limited mainly to that obtainable from wavelength discrimination and sun incidence angle changes. The results of

Manuscript received February 7, 1985; revised May 28, 1985. This work was supported by the University of Nebraska Agricultural Experiment Station, by the NASA Fundamental Research Program under Grant NAG52777, by the NASA Graduate Researchers Program under Grant NGT 28004-801, and by the NASA Land Resources Program Multiple-Linear Array Technology Development Program under Contract S19583-D.

J. M. Norman and E. A. Walter are with the Department of Agronomy, University of Nebraska, Lincoln, NE 68583.

J. M. Welles is with LI-Cor, Inc., Lincoln, NE. recent studies suggest that multiple view angles may be used to great advantage in estimating the structure and characteristics of vegetation [1]. The relation between view angle and surface characteristics is only beginning to be understood. Clearly, from a remote-sensing perspective, observing a soil or vegetation surface from a range of view angles will improve our chances of extracting such useful information as soil roughness or canopy architecture. Unfortunately, this additional information costs more to obtain, and considerable research remains to be done to determine whether the additional benefit is worth the additional cost.

The objective of this paper is to illustrate how some of the most important surface properties affect the brightness of leaves, canopies, and soils when they are viewed from various directions..

Throughout this paper we will use the term bidirectional reflectance distribution function (BRDF); therefore, some discussion of this concept seems appropriate in this introduction. The directional dependence of surface reflectance on viewing angle can be characterized by comparing the radiance of the actual surface to the radiance of an ideal or Lambertian surface. If the instrument of interest has a very small field of view and the surface being viewed is very large, then the brightness of a Lambertian surface is independent of view angle. In general, for non-Lambertian surfaces, this distribution of apparent surface reflectance factors with view angle depends also on the source incidence angle. Since two directions are involved (source direction and view direction), the term bidirectional reflectance distribution function (BRDF) is used to describe the normalized surface brightness as a function of source-incidence and view angles [2]. BRDF has dimensions of reciprocal steradians and values can range between zero and infinity. Slater [3] suggests that "... the BRDF relates the directional radiance of the surface to the directional irradiance and directional source radiance, respectively."

The following sections of this paper represent our attempt to illustrate the important features that cause observed BRDF's.

\section{BARE SOIL}

The BRDF for bare soils usually is highly non-Lambertian. In fact, the soil BRDF can be more non-Lambertian than the BRDF from most vegetated surfaces [4].

A very simple model can be used to gain much insight 


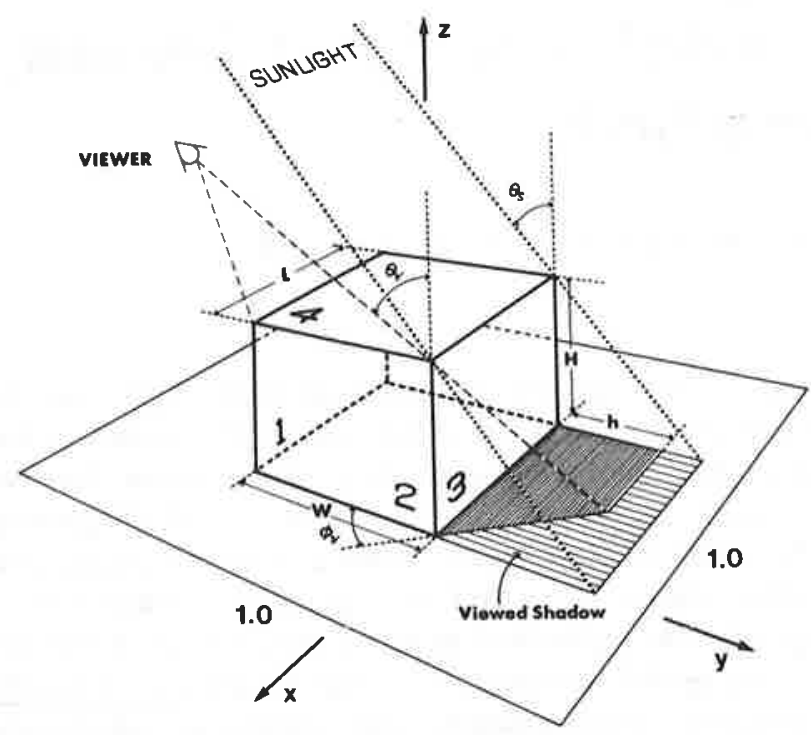

Fig. 1. Sketch of soil block and projected shadow that forms the basis for soil BRDF model.

into the dependence of soil brightness on view angle by considering shadowing effects of an idealized soil aggregate. Consider this soil aggregate to be a single block of length $L$ (perpendicular to the sun azimuth direction), width $W$, and height $H$ that is centered on a square lot area of unity dimensions, which is composed of the same smooth soil material as the block (Fig. 1). In addition, all surfaces are assumed Lambertian so that the predicted soil BRDF arises from shading effects of larger particles or aggregates rather than scattering properties of basic soil particles of silt or clay. If azimuth angles are referenced to the $-y$ axis (Fig. 1) and the azimuth of the sun is always zero for simplicity, then for a given sun zenith angle $\theta_{s}$, the soil BRDF can be estimated from the relative contributions of sunlit and shaded areas to the scene brightness for a wide range of view directions $\left(\theta_{v}, \phi_{v}\right)$. This analysis assumes the observer has a small field of view and is relatively far from the block. Assuming the direct-beam flux density to be unity on a surface perpendicular to the sun's rays and a sky diffuse illumination of $d$, the scene brightness can be estimated by combining the brightness of each surface with the area of that surface projected onto the horizontal. In the case of the shadow projected by the block, only that portion of the shadow that can be seen is included in the scene brightness estimate. The following equations describe the horizontally projected areas of the block faces and the shadow that are viewed by an observer:

$$
\text { If } \phi_{v} \geq 90 \quad \begin{aligned}
A_{1} & =0 \\
A_{2} & =H W \tan \theta_{v} \sin \phi_{v} \\
A_{3} & =H L \tan \theta_{v}\left|\cos \phi_{v}\right| \\
A_{4} & =L W \\
A_{s} & =L H \tan \theta_{s} .
\end{aligned}
$$

If $\phi_{v}<90$

$$
\begin{aligned}
A_{1}= & H L \tan \theta_{v} \cos \phi_{v} \\
A_{2}= & H W \tan \theta_{v} \sin \phi_{v} \\
A_{3}= & 0 \\
A_{4}= & L W \\
A_{s}= & \left\{\begin{array}{c}
\frac{1}{2} h^{2} \tan \phi_{v}+L\left(H \tan \theta_{a}-h\right), \\
h \leq H \tan \theta_{s} \\
\frac{1}{2}\left(H \tan \theta_{s}\right)^{2} \tan \phi_{v}, \\
h>H \tan \theta_{s}, \phi_{v}<\phi_{c} \\
L H \tan \theta_{s}-\frac{1}{2} L^{2} \cot \phi_{v}, \\
h>H \tan \theta_{s}, \phi_{v} \geq \phi_{c}
\end{array}\right. \\
h= & H \tan \theta_{v} \cos \phi_{v} \\
\phi_{c}= & \tan ^{-1}\left[L /\left(H \tan \theta_{s}\right)\right]
\end{aligned}
$$

where $A_{1}$ is the area of the sunlit face of the block projected onto the horizontal, $A_{3}$ is the area of the shaded face adjacent to the shadow of the block projected onto the horizontal, and $A_{2}$ is the horizontal projection of the block surface connecting faces 1 and 3 . Surface $A_{2}$ is always shaded. $A_{4}$ is the top surface, and $A_{s}$ is the area of the block's shadow on the horizontal that can be "seen" (Fig. 1). This shadow area $A_{s}$ is kept separate from the shaded block surfaces so that the block could be assigned a different reflectance if desired. The alert reader will realize that horizontal projections of vertical surfaces can become very large when zenith angles of the sun or viewer are large. Therefore, without restrictions, the horizontally projected areas for the shadow and block faces may exceed the lot area of unity. To avoid this eventuality two restrictions are invoked:

$$
\text { Width of shadow }=H \tan \theta_{s} \leq 1-W
$$

Viewed area of shadow $=A_{s} \leq 1-A_{1}-A_{2}-A_{3}$.

If either $H \tan \theta_{s}$ or $A_{s}$ exceed these limits, the limiting values are used. The rationale for invoking these limits is that as sun or view zenith angles increase, the likelihood of the shadow being obscurred by projections of other surfaces increases. Also, shadows that extend beyond the lot drea are ignored. Without these restrictions (1) is appropriate only over a relatively narrow range of zenith angles. Since the block is centered on the lot area, these restrictions imply a "folding" of projections: that is, a projection that would extend beyond one side is assumed to cover the lot area on the opposite side. The second restriction also implies that the top block surface can be obscured by shadow; although this may not be possible physically, a better fit of model to measurement results from this constraint.

The BRDF for the part of this simplified scene can be calculated if the relative irradiance on each surface is 


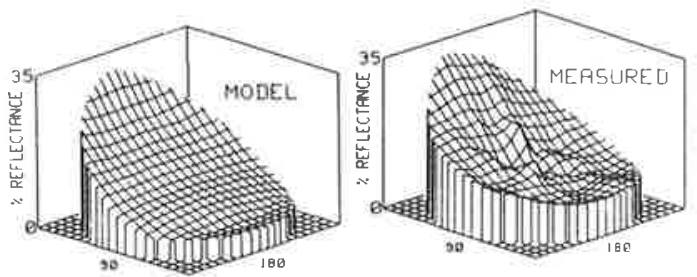

(a)

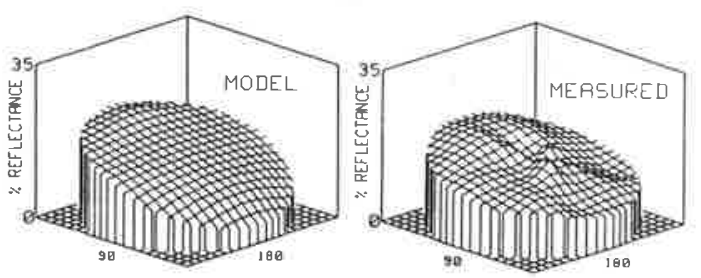

(b)

Fig. 2. Soil BRDF predicted from simple model $(H=0.4, L=0.85, W=$ $0.1, \sigma=0.67$ ) and measured for a rough plowed soil with furrows and $15-\mathrm{cm}$ clods for a solar zenith angle of (a) $63^{\circ}$ and (b) $28^{\circ}$. The rms difference between measured and modeled is 2.2 percent for (a) and 1.47 percent for (b) in reflectance units. Maximum view angle is $60^{\circ}$ (outer rim), center is nadir. Sun is at $0^{\circ}$ azimuth.

known. Assuming the relative direct irradiance on a plane perpendicular to the direction of the sun to be unity and diffuse irradiance to be $d$, the relative apparent radiance of each block surface is as follows:

$$
\begin{aligned}
& R_{1} \simeq \sin \theta_{s}+d \\
& R_{2}=R_{3}=R_{s}=d \\
& R_{4}=\cos \theta_{s}+d .
\end{aligned}
$$

Of course these are simplified expressions because diffuse illumination of vertical surfaces would depend on whether adjacent surfaces were sunlit or shaded. A relative BRDF can be calculated by summing the contributions of all the block faces, the block shadow, and the background lot area

$$
\begin{aligned}
R_{x}= & \left(1-A_{1}-A_{2}-A_{3}-A_{s}\right) R_{4}+A_{1} R_{1} \\
& +A_{2} R_{2}+A_{3} R_{3}+A_{s} R_{s} .
\end{aligned}
$$

The true soil BRDF can be obtained by multiplying (3) by the ratio of a measured nadir soil reflectance $\left(\rho_{n}\right)$ and the nadir reflectance $\left(R_{n}\right)$ calculated from (3)

$$
R_{n}=\left(1-A_{s}\right) R_{4}+A_{s} R_{s} .
$$

Equations (1)-(3) represent a very simple model of the BRDF for a soil which captures many of the important features present in measurements (Fig. 2(a),(b)). The measured distributions in Fig. 2(a),(b) increase with increasing zenith view angle at the $180^{\circ}$ azimuth, and the model results do not do this. Two possible explanations for this increase are as follows: 1) The collimator on the sensor that was used to measure the soil BRDF may have allowed some stray light to reach the detector when pointing toward the sun at higher zenith view angles. 2) Some soil surfaces may exhibit a significant specular reflection component from the irregular surfaces. Since the orientation of the block remains fixed relative to the azimuth of the sun, the predicted BRDF is always symmetric about

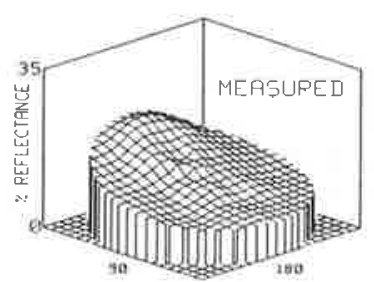

(a)

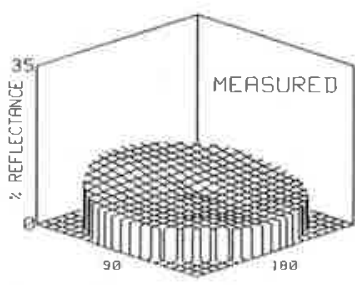

(b)
Fig. 3. Measured soil BRDF for (a) medium roughness soil (2- to $5-\mathrm{cm}$ clods from multiple tillages) and (b) a smooth soil (about $0.5-\mathrm{cm}$ gravel) with a solar zenith angle of $43^{\circ}$. Model fits to these data were (a) $H=$ $0.4, L=0.85, W=0.1, \sigma=0.67$ and (b) $H=0.4, L=0.6, W=0.1$, $\sigma=0.6$ and the rms reflectance differences between model and data were 1.1 percent. Maximum view zenith angle is $60^{\circ}$ (outer rim). Sun is at $0^{\circ}$ azimuth.

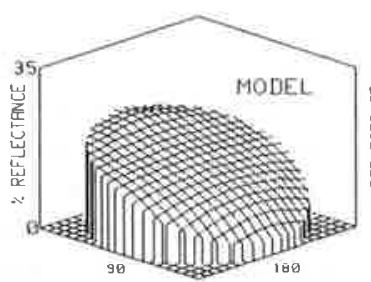

(a)

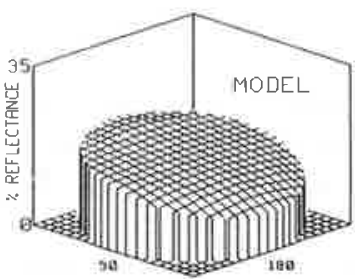

(b)
Fig. 4. Dependence of modeled BRDF on fraction of total radiation that is diffuse: (a) 5 percent diffuse, (b) 30 percent diffuse. Maximum view zenith angle is $60^{\circ}$ (outer rim). Sun is at $0^{\circ}$ azimuth.

the principal plane. (The principal plane is a vertical plane containing the spot being viewed and the sun.) The measured distributions in Fig. 2 contain "lumpiness" near the centers; that is characteristic of measured soil and canopy BRDF's because the small footprint on the surface, which is associated with near-nadir viewing angles, emphasizes surface variability. Soil roughness may affect the soil BRDF; in general, a smoother soil tends to have a smoother BRDF. A simple relative roughness parameter is given by

$$
\sigma=[H L(1-W)]^{1 / 3} .
$$

One might wonder why width $(W)$ enters in a roughness relation as in (5). Capturing the importance of three length dimensions on BRDF with a single number is impossible; however, at least (5) yields a $\sigma$ that tends to increase as $H$, $L$, and $W$ are varied to simulate rougher soils. With the model a large value of $\sigma$ might be 1.0 and as $\sigma$ gets small the soil becomes Lambertian and the BRDF becomes "flat." From this simple model, however, the relative size of elements compared with spacing is important and not the absolute size of soil aggregates. That is, the relative size of observed sunlit and shaded regions is most important. It is not too surprising then that very different surfaces can have similar bidirectional reflectance distribution functions (Fig. 3).

The soil BRDF will depend on wavelength because of the spectral properies of the material that make up the aggregates and because of the illumination level of shaded areas. At visible wavelengths the sky brightness is much greater than at near-infrared (NIR) wavelengths; therefore, shaded regions are likely to be brighter in the visible 
and variations in the bidirectional reflectance factor with view angle appropriately less (Fig. 4). Below a canopy, however, diffuse levels may be much greater in the near infrared than in the visible because of the scattering properties of leaves so that care must be used in combining soil and canopy BRDF's.

The shape of soil aggregates may also have an effect on the BRDF, but in practice this effect is not likely to be very dramatic. The measurements in Fig. 2(a) are from a field with deep furrows. If the model is altered to simulate long thin ridges perpendicular to the sun direction $(L=1$ and face 2 disappears), the results are very similar to those predicted in Fig. 2(a).

\section{Individual LeAves}

A remote observation of any plant canopy derives from the collective effect of all the individual component parts including leaves, stems, flowers, and, of course, the soil background when the canopy is sparse. Therefore, canopy remote signatures can best be understood by characterizing at least the most important components. The most important radiative components in live vegetation are leaves, and their radiative properties depend on their surface properties, cellular structure, and composition. Unfortunately the dependence of leaf reflectance or transmittance on leaf make-up is exceedingly complex, and little is known beyond the general effects of pigment absorption in the visible air-water interface scattering in the nearinfrared and water absorption in the middle infrared [5], [6]. Many measurements of leaf spectral properties are available over a wide range of wavelengths [7]. However, most of these measurements are made with near-normalincidence sources and integrating spheres that collect all of the radiation eminating from the leaf. Few measurements have ever been made of the BRDF of leaves. Breece and Holmes [8] made measurements on corn and soybean leaves at source incidence angles of $0^{\circ}, 30^{\circ}$, and $60^{\circ}$ and view angles in the principal plane. These measurement results show the very strong dependence of BRDF on source incidence angle and wavelength. Some estimates of specular reflectance have been made from polarization measurements at Brewster's angle [9]. The results of these measurements on many species indicate that the specularly reflected radiation may vary from 10 percent (soybean) to excess of 50 percent (corn and sorghum) in visible wavelengths. Obviously, surface characteristics, such as the amount of waxes, are important to the amount of specular reflectance.

The BRDF and bidirectional transmittance distribution function (BTDF) for corn and soybean leaves are shown in Fig. 5 for a source incidence angle of $45^{\circ}$. Clearly the apparent brightness of a leaf depends very strongly on the angle from which the leaf is viewed. The enhanced reflection from view angles near $180^{\circ}$ (looking toward the source) suggests specular reflection from an irregular leaf surface. Clearly from Fig. 5, the leaf BRDF depends strongly on wavelength. Although not shown, the leaf

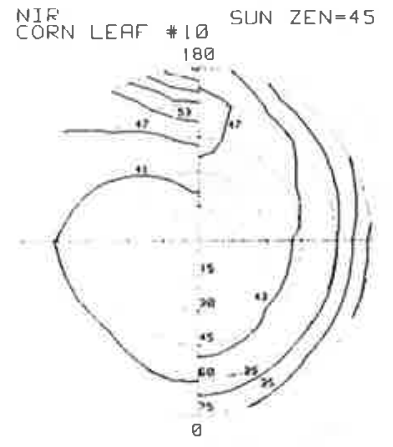

REFLECTANCE TRANSMITTANCE

(a)

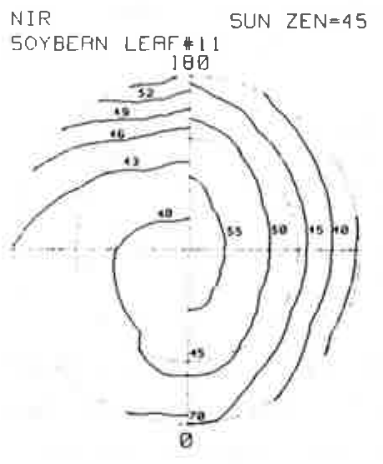

REFLECTANCE TRANSMITTANCE

(c)

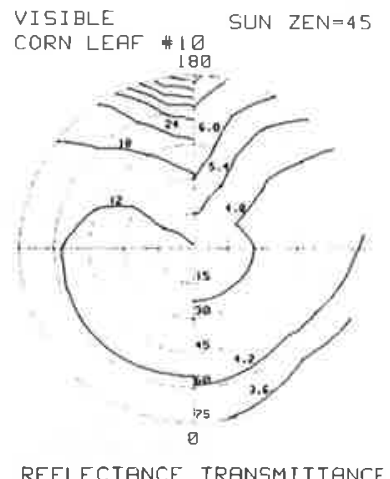

(b)

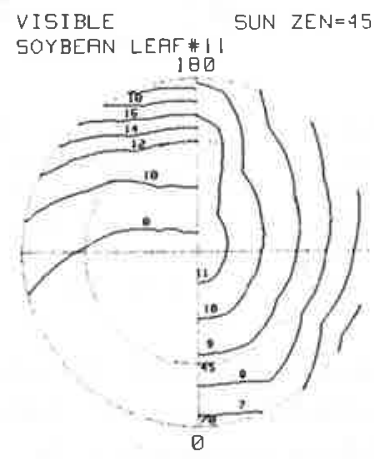

REFLECTANCE TRRNSMITTANCE

(d)
Fig. 5. Leaf BRDF and bidirectional transmittance distribution function at a source incidence angle at $45^{\circ}$ for (a) corn at near infrared wavelengths, (b) corn at visible wavelengths, (c) soybean at near infrared wavelengths, and $(d)$ soybean at visible wavelengths. Source at $0^{\circ}$ azimuth.

BRDF also has a marked dependence on source incidence angle.

All models of radiative transfer in vegetation require leaf spectral properties to be known. Usually leaves are assumed to be Lambertian and near-normal-incidence integrating sphere measurements are used to estimate the leaf hemispherical reflectance or transmittance. Few leaves in most canopies experience direct sunlight at normal incidence. A more representative incidence angle for individual leaves in a canopy would be $45^{\circ}$ and in some canopies, such as corn, $60^{\circ}$ would be more reasonable. Unfortunately, the hemispherical leaf reflectance depends on source incidence angle, and normal incidence values of reflectance tend to be too low and transmittance too high compared with other incidence angles (Table I). In corn and soybeans, the errors that occur from using normal-incidence leaf properties instead of mean-incidenceangle properties can be significant.

\section{Plant Canopies}

Radiative transfer in vegetation has been studied for many years because of the impact of this radiation on plant production and water use [10], [11]. Past research has emphasized radiation penetration into canopies; however, the same simple and approximate geometric approaches can be used to model plant canopy BRDF's.

The BRDF for a vegetation canopy is influenced by many factors including the following: 1) zenith angle of 
TABLE I

Hemispherical Reflectance and Transmittance of Fleld-Grown Corn and Greenhouse-Grown Soybean Calculated by Integrating THE RESUlts of LEaf BRDF MEasurments

\begin{tabular}{|c|c|c|c|c|c|c|}
\hline \multirow[b]{2}{*}{ Crop } & \multirow{2}{*}{$\begin{array}{l}\text { Incidence } \\
\text { Angle }\end{array}$} & \multicolumn{2}{|c|}{ Visible } & \multicolumn{3}{|c|}{ NIR } \\
\hline & & Refl & (\%) Trans (\%) & $\operatorname{Refl}$ & $(\%) \mathrm{T}$ & Trans (\%) \\
\hline \multirow[t]{4}{*}{ Corn } & 0 & 7.7 & 3.9 & 42 & & 53 \\
\hline & 20 & 9.1 & 4.2 & 42 & & 48 \\
\hline & 45 & 9.3 & 3.4 & 42 & & 44 \\
\hline & 70 & 14.2 & 3.9 & 37 & & 36 \\
\hline \multirow[t]{4}{*}{ Soybean } & 0 & 8.3 & 7.0 & 40 & & 48 \\
\hline & 20 & 9.3 & 7.5 & 42 & & 47 \\
\hline & 45 & 9.6 & 8.2 & 44 & & 45 \\
\hline & 70 & 16.1 & 7.0 & 55 & & 38 \\
\hline
\end{tabular}

(The results for normal incidence are from integrating sphere measurements. For corn, the mid-rib of the leaf was oriented perpendicular to the direction of the light source.)

the sun; 2) ratio of direct beam to sky diffuse illumination; 3) distribution of leaf angles in the canopy; 4) leaf BRDF and BTDF of top and bottom leaf surfaces; 5) soil BRDF; 6) leaf area per unit of ground area in the canopy (leaf area index (LAI); 7) horizontal and vertical distribution of leaf position such as individual trees, row structures, or uniform cover; 8) distribution, spectral properties, and amount of component structures other than leaves such as stems, flowers, or heads, and 9) wavelength. Kimes [12] discusses many of these factors and presents numerous BRDF measuremens for various canopies and a bare soil. The approach to be described here first considers radiation penetration into a vegetation canopy in an average sense, then the canopy BRDF is obtained from a combination of this average solution and viewing angle considerations.

A simple model of radiation penetration into vegetation is based on dividing the canopy into $N$ thin layers each of equal leaf-area-index increment $\Delta F$ [11]. For simplicity in this derivation we can assume each layer to have the same reflectance $\left(R_{L}\right)$ and transmittance $\left(T_{L}\right)$. These layer properties can be related to leaf reflectance and transmittance and canopy geometry. Again for simplicity in this derivation, assume leaves to be randomly positioned in the horizontal with any leaf angle distribution $[g(\alpha, \beta)]$. If the fraction of direct beam radiation intercepted by a canopy layer of LAI increment $\Delta F$ is given by $I_{B}\left(\theta_{s}, \phi_{s}\right)$

$$
I_{B}\left(\theta_{s}, \phi_{s}\right)=1-\exp \left(-k_{B} \Delta F / \cos \theta_{s}\right)
$$

then the direct beam radiation that would be reflected or transmitted through leaves in a layer can be defined in terms of leaf reflectance $\left(\rho_{l}\right)$ and transmittance $\left(\tau_{l}\right)$ as

$$
\begin{aligned}
& R_{L, B}=I\left(\theta_{s}, \phi_{s}\right) \rho_{l} \\
& T_{L, B}=I\left(\theta_{s}, \phi_{s}\right) \tau_{l}
\end{aligned}
$$

where $k_{B}$ is the extinction coefficient for direct beam radiation from a solar zenith angle $\theta_{s}$ and azimuth angle $\phi_{s}$ given by

$$
k_{B}=\int_{0}^{\pi / 2} \int_{0}^{2 \pi} g(\alpha, \beta)\left|\cos \delta_{s}\right| d_{\beta} d_{\alpha} / 2 \pi
$$

where

$$
\begin{aligned}
\left|\cos \delta_{s}\right|= & \mid \cos \theta_{s} \cos \alpha \\
& +\sin \theta_{s} \sin \alpha \cos \left(\beta-\phi_{s}\right) \mid
\end{aligned}
$$

with $\alpha$ being leaf inclination angle to the horizontal and $\beta$ being leaf azimuth angle. Essentially $k_{B}$ is the fraction of the entire canopy leaf area projected in the direction of the sun and $\cos \left(\delta_{s}\right)$ is the projection of that single leaf element, which is inclined at the angle $\alpha$ and oriented at $\beta$ in the direction of the sun. Several simplified distributions are useful such as the spherical $\left(k_{B}=0.5\right)$, horizontal $\left(k_{B}=\cos \theta_{s}\right)$, vertically inclined with azimuthal symmetry $\left(k_{B}=2 \sin \theta_{s} / \pi\right)$, and even heliotropic [10]. General leaf angle distributions with azimuthal symmetry can be represented by two coefficients with the beta distribution [13].

If all of the diffuse angular distributions can be assumed isotropic, then the diffuse interception factor for any layer can be obtained from the integral of (6)

$$
I_{d}=\int_{0}^{\pi / 2} \int_{0}^{2 \pi} I_{B}(\theta, \phi) \sin \theta \cos \theta d \theta d \phi / \pi
$$

The diffuse layer reflectance and transmittance can be defined as

$$
\begin{aligned}
& R_{L, d}=I_{d} \rho_{l} \\
& T_{L, d}=I_{d} \tau_{l}+\left(1-I_{d}\right)
\end{aligned}
$$

where $1-I_{d}$ is the fraction of diffuse radiation that passes through the layer without intercepting any leaves. An interesting note is that the leaf properties for diffuse radiation (11) need not be the same as leaf properties for direct beam radiation (7). In fact leaf reflectance and transmittance for direct beam radiation depend on the sun incidence angle with the leaf normal.

Using the preceding equations a set of layer equations can be written for scattered radiation in the canopy. If the top canopy layer is denoted by $N$ and the soil surface by zero, and $f_{B}$ is the fraction of the radiation above the canopy that is direct beam, then the downward diffuse flux density above an intermediate layer $j\left(D_{j}\right)$ is

$$
D_{j}=\left(1-f_{B}\right)\left(D_{j+1} T_{L, d}+U_{j} R_{L, d}\right)+S_{j+1} T_{L, B}
$$

where $S_{j+1} T_{L, B}$ is the direct beam source of downward diffuse radiation that arises from interception in layer $j+$ 1 and $S_{j+1}$ is given by

$$
S_{j+1}=f_{B} D_{N} \exp \left(-k_{B} F_{j+1} / \cos \theta_{s}\right)
$$

where $F_{j+1}$ is the leaf area index above layer $j+1$. The upward flux density above layer $j+1$ is given by

$U_{j+1}=\left(1-f_{B}\right)\left(U_{j} T_{L, d}+D_{j+1} R_{L, d}\right)+S_{j+1} R_{L, B}$. 
Equations (12)-(14) can be solved in any of several ways using boundary conditions of soil reflectance at the bottom $\left(U_{0} / D_{0}=\rho_{\text {soil }}\right)$ and $D_{N}=1$ at the top [11], [14]. The result is all the upward and downward diffuse fluxes in the canopy.

Several assumptions have been made in this derivation such as isotropic diffuse radiation angular distributions and Lambertian leaves. Clearly these assumptions are not necessary with a numerical solution of the preceding equations. The diffuse angular distribution (canopy BTDF) in the canopy has been shown to have a very small effect on radiation penetration into vegetation under clear sky conditions [14], and this BTDF is likely to have an even smaller effect on the canopy BRDF. The non-Lambertian leaf effect is not negligible and has been incorporated in the model Cupid using data for leaves such as that in Table I and Fig. 5. Results from the model Cupid will be presented later. A further assumption of random leaf positioning also has been removed [15], [16].

The previous model for radiation penetration into vegetation can be combined with an analysis of viewing effects to provide equations that predict canopy BRDF. The previous equations are particularly useful for obtaining the diffuse and direct beam irradiation on a leaf. However, the radiance in a particular viewing direction depends not only on the direct and diffuse irradiance on leaves, but also on the relationship between sun and view angles. The radiance $\left[R_{c}\left(\theta_{v}, \phi_{v}\right)\right]$ from the canopy in a particular viewing direction $\left(\theta_{v}, \phi_{v}\right)$ can be approximated as follows, if each layer in the canopy is of equal thickness $\Delta F$ :

$$
\begin{aligned}
R_{c}\left(\theta_{v}, \phi_{v}\right)= & \sum_{j=1}^{N} \int_{\alpha} \int_{\beta} g(\alpha, \beta) \\
& \cdot\left\{\left[\left|f_{s}\right| f_{B} D_{N} \rho_{l}^{*} / \cos \theta_{s}+E_{j}(\alpha)\right] C_{j}\right. \\
& \left.+E_{j}(\alpha)\left(1-C_{j}\right)\right\}\left|f_{v}\right| W_{j} d \beta d \alpha
\end{aligned}
$$

where

$$
\begin{aligned}
& g(\alpha, \beta) \text { is the fraction of leaf area inclined at } \alpha \text { and } \\
& \text { oriented at } \beta \text {. } \\
& f_{s} \quad=\cos \delta_{s}(\operatorname{see}(9)) \text {. } \\
& f_{v} \quad=\cos \delta_{v}\left((9) \text { with } \theta_{v}, \phi_{v} \text { replacing } \theta_{s}, \phi_{s}\right) \text {. } \\
& f_{B} \quad \text { is the fraction of radiation above canopy that } \\
& \text { is direct beam. } \\
& D_{N} \quad \text { is the total irradiance above canopy on hori- } \\
& \text { zontal. } \\
& =p_{1} D_{j} \rho_{l}+p_{2} D_{j} \tau_{l}+p_{1} U_{j-1} \tau_{l}+p_{2} U_{j-1} \rho_{l}, \\
& \text { if } f_{v} \geq 0 \text {. } \\
& =p_{1} D_{j} \tau_{l}+p_{2} D_{j} \rho_{l}+p_{1} U_{j-1} \rho_{l}+p_{2} U_{j-1} \tau_{l}, \\
& \text { if } f_{v}<0 \text {. }
\end{aligned}
$$

tropic diffuse radiation impinging on the top (bottom) of a leaf inclined at $\alpha$ [14].

$=(1+\cos \alpha) / 2$.

$p_{2} \quad$ is the fraction of downward (upward) isotropic diffuse radiation impinging on the bottom (top) of leaf inclined at $\alpha$ [14].

$=(1-\cos \alpha) / 2$.

$\rho_{l}, \tau_{l} \quad$ is the leaf reflectance and transmittance; in general both $\rho_{l}$ and $\tau_{l}$ may be different for direct beam and diffuse radiation.

$\rho_{l}^{*} \quad$ is the reflectance of the top of the leaf when the sun shines on the top of the leaf and the viewer sees the top of the leaf $\left(f_{s}>0, f_{v}>\right.$ $0)$. The leaf reflectance of the bottom of the leaf would be appropriate if $f_{s}<0$ and $f_{v}<$ 0 . If $f_{s}>0, f_{v}<0$ or $f_{v}>0, f_{s}<0$ then the leaf transmittance is used in this term.

$D_{j}, U_{j} \quad$ are the downward and upward diffuse flux density above layer $j$, respectively.

$W_{j} \quad$ is the factor that weights the contribution of various layers to the view from a particular direction; using (6) and replacing $B$ and $s$ subscripts referring to the sun by $v$ subscripts referring to the view, $=\exp \left(-k_{v} F_{j} / \cos \theta_{v}\right) I_{v}\left(\theta_{v}, \phi_{v}\right)$.

$C_{j} \quad$ is the fraction of leaf area in layer $j$ that is sunlit.

$$
=\exp \left(-k_{B} F_{j} / \cos \theta_{s}\right) I_{B}\left(\theta_{s}, \phi_{s}\right) \cos \theta_{s} /
$$
$\left(k_{B} \Delta F\right)$.

$F_{j} \quad$ is the leaf area index above layer $j$.

$k_{B}, k_{v} \quad$ is the extinction coefficient for direct beam radiation (8), and extinction coefficient for view ((8) with $\delta_{v}$ replacing $\delta_{s}$ ).

The weighting factors $\left|f_{v}\right|$ and $W_{j}$ must be normalized to unity. The contribution of the soil layer can be derived from a combination of the soil block model presented previously and the canopy equations above as follows:

$$
\begin{aligned}
R_{g}\left(\theta_{v}, \phi_{v}\right)= & {\left[\left(f_{B} D_{N} / \cos \theta_{s}\right) R_{x}\left(\rho_{n} C_{0} / R_{n}\right)\right.} \\
& \left.+D_{0} \rho_{\text {hem }}\left(1-C_{0}\right)\right] W_{0}
\end{aligned}
$$

where $\rho_{n}$ is the nadir-view measured soil reflectance, $R_{n}$ is from (4), $\rho_{\text {hem }}$ is the soil hemispherical reflectance from the integral of (3), $R_{x}$ is from (3), and $C_{0}$ is the fraction of sunlit soil given by

$$
C_{0}=\exp \left(-k_{B} F_{0} / \cos \theta_{s}\right)
$$

and $W_{0}$ is a weighting factor accounting for the amount of soil contributing to the "view" given by

$$
W_{0}=1-\sum_{j=1}^{N} W_{j} \text {. }
$$

The $F_{0}$ is the canopy leaf area index. The diffuse contribution $d$, used in (2), is given by

$$
d=D_{0} \cos \theta_{s} /\left(f_{B} D_{N}\right) .
$$

If the measured soil reflectance that is used as a boundary 


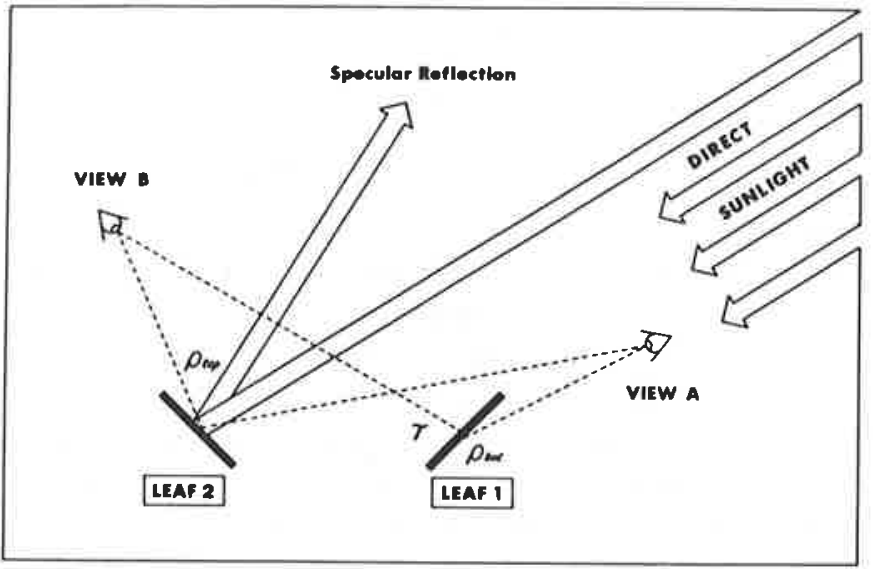

Fig. 6. Illustration of canopy geometry that contributes to the observed canopy BRDF.

condition is a hemispherical measurement, then $\rho_{n}$ must be computed from this measured hemispherical value and the integral of (3). Equations (15) and (16) are summed to yield the final BRDF of the canopy plus soil system.

All of the preceding equations for canopy and soil BRDF are appropriate for a single narrow-wavelength band. Clearly more generality can be obtained with the numerical evaluation of (12) than is shown in that equation. For example, the leaf angle distribution may depend on depth, or the layers need not be in equal increments of leaf area index, or specular reflectance equations could be incorporated. Furthermore, the angular distribution (or BTDF) of radiation within the canopy could be incorporated, but under clear-sky conditions this is a lot of computation with marginal improvement in predictions of BRDF.

The preceding equations represent one approach to modeling homogeneous-vegetation-canopy BRDF. Other models have been developed such as that of Suits [17]. Although the model of Suits is relatively uncomplicated and does capture the character of canopy BRDF's, it does not adequately represent the leaf angle distribution or include leaf BRDF effects or soil BRDF. This model was extended to include the leaf angle distribution in greater detail and is referred to as the SAIL model [18].

The various canopy BRDF models attempt to consider the dominant effect of leaf angle distribution on the radiance distribution. The presence of the leaf angle distribution $g(\alpha, \beta)$ in (15) along with sun and view projections $\left(f_{s}, f_{v}\right)$ shows clearly the importance of leaf angles. The origin of some of the terms in (15) can be illustrated by a very simple example. Consider two flat leaves in the principal plane, each inclined at $45^{\circ}$ with a sun zenith angle of $50^{\circ}$ (Fig. 6). Leaf 2 is nearly perpendicular to the sun and thus is brightly illuminated while leaf 1 is nearly parallel to the sun and only dimly lit by the direct beam. An observer with view $A$ in Fig. 6 (sun behind observer) will "see" the bright leaves well and will not "see" the dimly lit leaves. Thus the scene appears bright. In the case of view $A$ in Fig. 6, the scene brightness is influenced by the reflectance of the bottom of leaf 1 and top of leaf 2 . An observer with view $B$ in Fig. 6 will "see" leaf 1 well,
HORIZONTRL VERTICAL

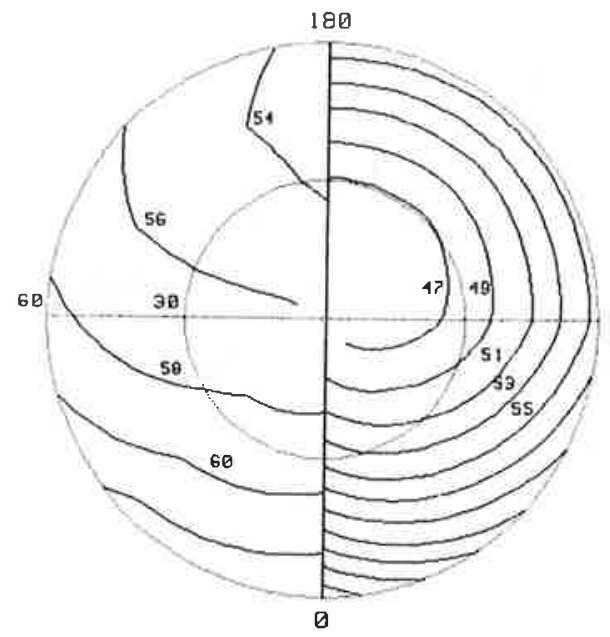

Fig. 7. Modeled canopy BRDF for a canopy with characteristics similar to a soybean canopy ( $\mathrm{LAI}=2.9$ ) but with an erectophile leaf orientation (mostly vertically oriented leaves) and planophile leaf orientation (mostly horizontally oriented leaves). Sun zenith is $63^{\circ}$ and sun azimuth is $0^{\circ}$.

which is dimly lit, and will not "see" much of leaf 2 , which is brightly lit. Therefore, from view $B$ the scene will appear darker. From view $B$ the transmittance of leaf 1 and top reflectance of leaf 2 will determine the scene brightness. Specular reflectance from leaf 1 and leaf 2 will not be observed at either view $A$ or view $B$. An actual canopy is composed of many such leaves with a wide range of azimuth and zenith angles. Equation (15) is a formal statement of the various projections and spectral properties, along with the implicit assumption of random leaf positioning, that are required to predict canopy BRDF. A comparison of the BRDF for a canopy of mainly vertically oriented leaves with a canopy of predominantly horizontal leaves is shown in Fig. 7 for a leaf area index of 3.0 and a random canopy. Clearly observations of canopy brightness may lead to indirect estimates of canopy architecture [1].

Heterogeneous vegetation canopies which may have widely spaced rows, clumped foliage, or individual trees have an additional effect that random canopies do not exhibit: the shadowing effect of subcanopies, such as individual trees or rows, on adjacent canopies or the soil. This shadowing effect is similar to the soil shadowing illustrated in Fig. 1 except that the block is a vegetation canopy. This is illustrated in Fig. 9, which contains the predicted BRDF from a three-dimensional ellipsoidal model [15] and measurements for a young corn canopy of LAI $=0.4$ (Fig. 8). When the canopy consists of well defined rows, symmetry of the BRDF about the principal plane may be lost unless the sun is parallel or perpendicular to the rows. The major influence of the soil can be seen in Fig. 9 at view azimuths near $180^{\circ}$ (looking back toward the sun) because the reflectance factor continues to decrease as the view zenith angle increases. More dense vegetation tends to have a minimum reflectance factor at slightly off-nadir with increasing reflectance factor as the view zenith angle increases. Another feature in the measured results in Fig. 9(a) is the clearly defined maximum 


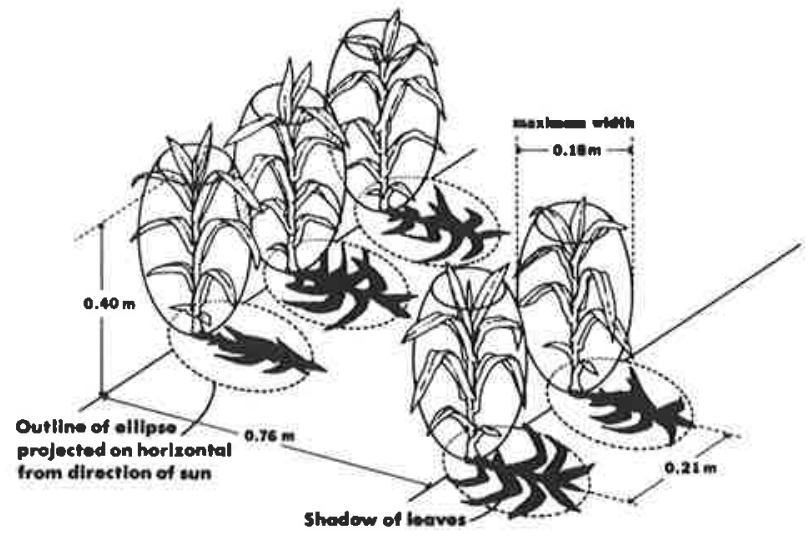

Fig. 8. Sketch of a corn canopy with LAI $=0.4$ including the ellipsoids used to approximate the canopy structure.

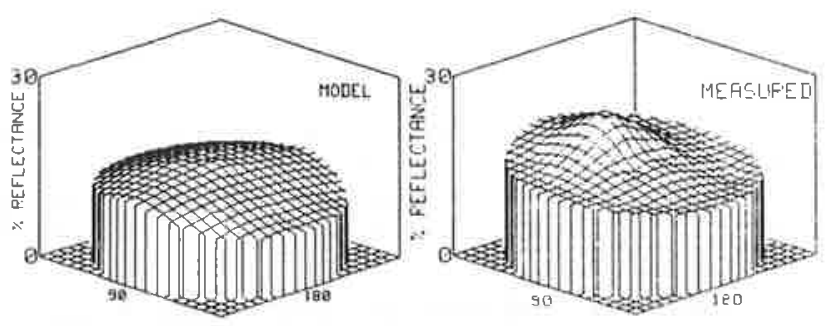

(a)
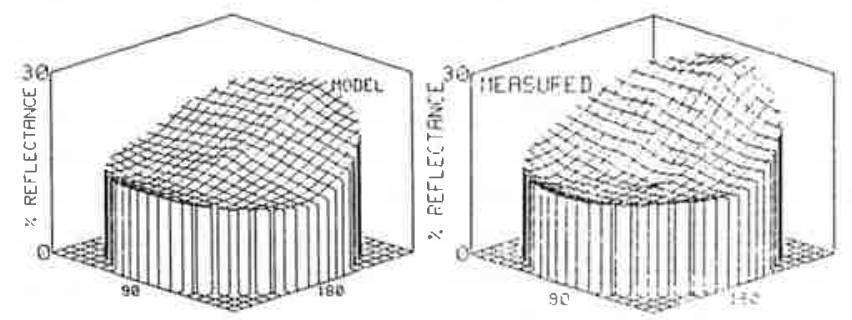

(b)

Fig. 9. Comparison of measurements on a corn canopy of LAI $=0.4$ having north-south rows with model predictions. (a) Solar azimuth of $7^{\circ}$ and solar zenith of $18^{\circ}$. (b) solar azimuth of $276^{\circ}$ and solar zenith of $47^{\circ}$. South is at $0^{\circ}$. Maximum view zenith angle is $60^{\circ}$ (outer rim), nadir view is at center.

when the sun is behind the observer. This feature is often termed the canopy "hot spot" and the equations presented in this paper do not adequately describe this feature.

\section{Summary}

The BRDF's of vegetation canopies and soils have common features that arise either from leaf projections and shading or shadowing from soil aggregates. The BRDF for individual leaves differs from that for canopies because of the greater importance of specular reflectance; furthermore, leaf surfaces are not smooth (relative to the wavelength of radiation) but they are much smoother than canopies so that canopies with specularly reflecting leaves are likely to have much different BRDF's than individual leaves in any event.

In remote sensing the ultimate benefit of models, such as those discussed in this paper, is to infer canopy features from radiance measurements. The canopy model discussed in this paper, which is named Cupid, has been inverted by N. Goel [1] to estimate leaf area index and mean leaf inclination angles. This, however, is not a simple inversion problem. The relatively complex surfaces that represent soil and canopy BRDF's can be fit with a threeparameter equation of the following form:

$$
R=a \theta_{v}^{2}+b \theta_{v} \cos \left(\phi_{v}-\phi_{s}\right)+c
$$

where $\theta_{v}$ is the view zenith angle, $\phi_{v}$ is the view azimuth, $\phi_{s}$ is the sun azimuth, and $a, b$, and $c$ are fitting constants that can be obtained from three or more directional reflectance estimates by exact fit or linear least squares [19]. Equation (20) has been used to fit vegetation and soil BRDF's over a range of wavelengths, solar zenith angles and leaf area indicies with rms reflectance errors of about 0.2 percent in the visible and 2 percent in the near infrared. Equations such as (20) are very useful in reducing the number of observations required to characterize the BRDF of natural surfaces. This may be essential if we are to use satellite observations to obtain surface features. Equation (20) is most reliable in representing the BRDF surface when the three observations are on the principal plane; for example, $\theta_{v}=0, \phi_{v}=0 ; \theta_{v}=30, \phi_{v}=0$; $\theta_{v}=30, \phi_{v}=180$ would be a reasonable triplet with a solar zenith angle of $35^{\circ}$. Walthall et al. fit (20) to predictions from the model Cupid and showed that the coefficients are "well behaved" with solar zenith angle and leaf area index. Thus models such as those presented in this paper may be useful in deriving simpler algorithms for use in operational remote sensing.

\section{ACKNOWLEDGMENT}

The soil BRDF measurements in Figs. 2 and 3 were provided by Prof. G. S. Campbell of Washington State University, and the corn BRDF measurements in Fig. 9 were kindly provided by Dr. J. Ranson, Laboratory for Applications to Remote Sensing, Purdue University.

\section{REFERENCES}

[1] N. S. Goel and R. L. Thompson, "Inversion of vegetation canopy reflectance models for estimating agronomic variables V: Estimation of LAI and average leaf angle using measured canopy reflectances," Remote Sensing Environ., vol. 16, pp. 69-85, 1984.

[2] F. E. Nicodemus, J. C. Richmond, J. J. Hsia, W. H. Venable, I. W. Ginsberg, and T. Liperis, "Geometrical considerations for reflectance nomenclature," National Bureau of Standards Rep.

[3] P. N. Slater, Remote Sensing: Optics and Optical Systems. Reading, MA: Addison-Wesley, 1980.

[4] F. D. Eaton and I. Dirmhirn, "Reflected irradiance indicatrices of natural surfaces and their effect on albedo," Appl. Opt., vol. 18, pp. 994-1008, 1979.

[5] H. W. Gausman, "Reflectance of leaf components," Remote Sensing Environ., vol. 6, pp. 1-9, 1977.

[6] H. W. Gausman, "Visible light reflectance, transmittance, and absorptance of differently pigmented cottom leaves," Remore Sensing Environ., vol. 13, pp. 233-238, 1982.

[7] H. W. Gausman and W. A. Allen, "Optical parameters of leaves of 30 plant species," Plant Physiol, , vol. 52, pp. 57-62, 1973.

[8] H. T. Breece, III, and R. A. Holmes, "Bidirectional scattering characteristics of healthy green soybean and corn leaves in vivo," Appl. Opt., vol. 10, pp. 119-127, 1981.

[9] L. Grant, C. S. T. Daughtry, and V. C. Vanderbilt, "Measurements of specularly reflected radiation from leaves," Purdue Univ., West Lafayette, IN, LARS Tech. Rep. 081583, 1983.

[10] J. M. Norman, "Radiative transfer in vegetation," in Heat and Mass Transfer in the Biosphere, D. A. deVries and N. H. Afgan, Eds. Washington, DC: Scripta Book Co., 1975, pp. 187-206. 
[11] J. M. Norman and G. S. Campbell, "Applications of a plant-environment model to problems in irrigation," in Advances in Irrigation, vol. 2, D. Hillel, Ed. New York: Academic, 1983, pp. 155-188.

[12] D. S. Kimes, "Dynamics of direction reflectance factor distributions for vegetation canopies," Appl. Opt., vol. 22, pp. 1364-1372, 1983.

[13] N. S. Goel and D. E. Strebel, "Simple beta distribution representation of leaf orientation in vegetation canopies," Agron. J., vol. 76, pp. 800-802, 1984.

[14] J. M. Norman and P. G. Jarvis, "Photosynthesis in Sitka Spruce, V. Radiation penetration theory and a test case," J. Appl. Ecology, vol. 12, pp. 839-878, 1975.

[15] J. M. Norman and J. M. Welles, "Radiative transfer in an array of canopies," Agron. J., vol. 75, pp. 481-488, 1983.

[16] D. S. Kimes and J. A. Kirchner, "Radiative transfer model for heterogeneous 3-D scenes," Appl. Opt., vol. 21, pp. 4119-4129, 1982.

[17] G. H. Suits, "The calculation of the directional reflectance of vegetative canopy," Remote Sensing Environ., vol. 2, pp. 175-182, 1972.

[18] W. Verhoef, "Light scattering by leaf layers with application to canopy reflectance modelling: The SAIL model," Remote Sensing Environ., vol. 16, pp. 125-141, 1984.

[19] C. L. Walthall, J. M. Norman, J. M. Welles, G. Campbell, and B. L. Blad, "A simple equation to approximate the bidirectional reflectance from vegetative canopies and bare soil surfaces," Appl. Opt., vol. 24, pp. 383-387, 1985.

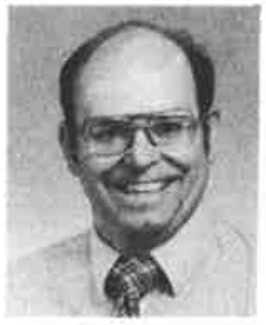

John M. Norman was born in Virginia, MN. He received the B.S. degree in physics and the M.S. degree in soil physics, both from the University of Minnesota, and the Ph.D. degree in soil physics from the University of Wisconsin, Madison, in 1971.

Following a 14-month fellowship in the Department of Botany at the University of Aberdeen, Scotland, working on radiation exchange in coniferous forests, he joined the Department of Meteorology, Pennsylvania State University, to work on measurements of atmospheric turbulence, information systems in meteorology, and radiation exchange in vegetation. In 1978, he joined the University of Nebraska as a Professor of Agronomy to work in the area of plant water relations. This biophysical research involves studies of the interactions between plants and their environments including measurements of soil, plant, and atmospheric characteristics and integrative modeling of the entire soil-plant-atmosphere system. Applications have included pest management, irrigation water use, and remote sensing.

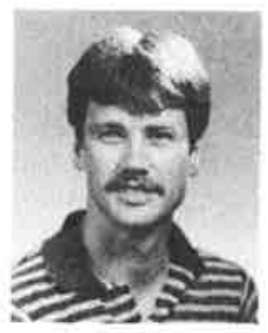

Jon M. Welles received the B.S. and M.S. degrees in meteorology from the Pennsylvania State University, University Park, PA. He is currently working toward the Ph.D. degree in agronomy at the University of Nebraska.

$\mathrm{He}$ is also currently employed by $\mathrm{Li}-\mathrm{COR}$, Inc., where he is involved in research and development.

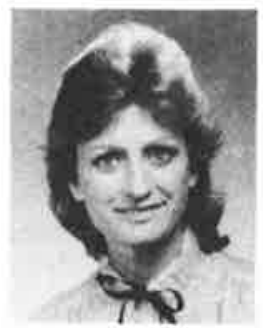

Elizabeth A. Walter received the B.S. and M.S. degrees from the University of Central Arkansas and Texas A\&M University in 1978 and 1981, respectively. She has worked at the Nebraska Remote Sensing Center and is working toward the $\mathrm{Ph}$.D. degree in agronomy at the University of Nebraska with an emphasis in remote sensing under the direction of the Center for Agricultural Meteorology and Climatology. 


